\title{
Productividad y calidad metodológica de artículos clínicos en cirugía oral y maxilofacial en Chile. Período 2001-2012*
}

\author{
Drs. PEDRO ARAVENA T. ${ }^{1,2}$, RICARDO CARTES-VELÁSQUEZ ${ }^{2,3,4}$, CARLOS MANTEROLA D. ${ }^{2,5}$
}

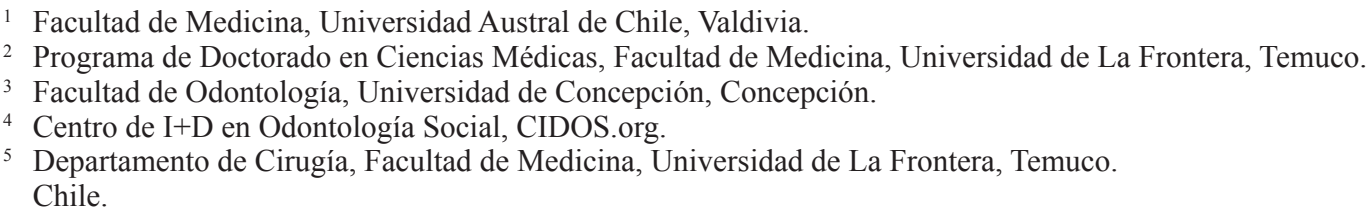

\section{Abstract \\ Productivity and methodological quality of clinical articles in oral and maxillofacial surgery in Chile. Period 2001-2012}

Introduction: Until now, there are no reports of quantity and quality of publications in oral and maxillofacial surgery (OMFS) conducted in Chile, which is why the aim of this study is to determine the productivity and methodological quality (MQ) of OMFS clinical articles in Chile in the period 2001-2012. Material and Methods: Bibliometric study. A systematic search in MEDLINE, Science Citation Index Expanded (Web of Science-ISI) and SciELO was conducted. Only clinical items related to OMFS made by Chilean authors were selected. Journal name, publication year, primary Chilean institution affiliated and specialty area were registered. Those articles related to surgical therapy were evaluated according to Sackett's level of evidence and MINCIR-therapy scale for MQ. Results are presented using descriptive statistics. Results: 68 articles were selected. Growth was observed in the number of publications, from $3(4.4 \%)$ in 2002 to $8(11.7 \%)$ in 2012, showing a peak (13 articles) in 2009. The most productive institution was Universidad de Chile $(\mathrm{n}=20)$. Majority of items are cases of surgical pathology. $91.2 \%$ of the articles observed had a type 4 level of evidence. The MQ average of analyzed articles was $9.9 \pm 4.0$ points, verifying only 4 items of good MQ (18 or more points). Conclusion: OMFS scientific production in Chile has increased during the last decade. However, the amount of articles of good level of evidence is low and MQ is poor.

Key words: "Surgery, Oral" [Mesh], "Bibliometrics" [Mesh], "Oral Surgical Procedures" [Mesh], "Evidence-Based Medicine" [Mesh].

*Recibido el 4 de marzo de 2013 y aceptado para publicacion el 1 de abril de 2013.

Los autores manifiestan no tener conflictos de interés ni recibir financiamiento para el desarrollo de esta investigación.

Correspondencia: Dr. Pedro Aravena T.

Rudloff 1640, Valdivia, Chile. Fax: (56-63) 221205

paravena@uach.cl 


\section{Resumen}

Introducción: A la fecha, no existen reportes referentes a cantidad y calidad de publicaciones en el área clínica de cirugía oral y maxilo-facial (COMF) realizadas en Chile; por ello, el objetivo de este estudio es determinar la productividad y calidad metodológica $(\mathrm{CM})$ de artículos clínicos de COMF en Chile en el período 2001-2012. Material y Método: Estudio bibliométrico. Se realizó una búsqueda sistemática en las bases de datos MEDLINE, Science Citation Index Expanded (Web of Science-ISI) y SciELO. Se seleccionaron sólo artículos clínicos atingentes a COMF realizados por autores chilenos. Se registró nombre de la revista, año de publicación, institución chilena afiliada y área de la especialidad. A aquellos artículos referentes a terapia quirúrgica se les aplicó la clasificación de niveles de evidencia Sackett y cols y la escala MINCIR-terapia para determinar CM. Los resultados se presentan mediante estadística descriptiva. Resultados: Se obtuvieron 68 artículos. Se observó un crecimiento en el número de publicaciones, desde $3(4,4 \%)$ el año 2002 a 8 $(11,7 \%)$ en el año 2012; con un incremento en 2009 (13 artículos). La institución con más publicaciones fue la Universidad de Chile $(\mathrm{n}=20)$. La mayoría de los artículos corresponden a casos de patología quirúrgica. Se observó un 91,2\% de artículos con nivel de evidencia tipo 4. La CM promedio de los artículos revisados fue de 9,9 \pm 4,1 puntos; verificándose sólo 4 artículos de buena CM (18 o más puntos). Conclusión: La producción científica en COMF en Chile ha aumentado en el transcurso de la última década. Sin embargo, la cantidad de artículos con buen nivel de evidencia y CM es baja.

Palabras clave: Cirugía oral, cirugía maxilofacial, bibliometría, medicina basada en la evidencia.

\section{Introducción}

Los análisis bibliométricos constituyen una herramienta de medición de la literatura que permiten determinar de forma crítica el desarrollo de la actividad científica ${ }^{1}$. Estos indicadores permiten valorar cantidad y calidad, así como el impacto de los artículos y las fuentes de información donde estos se publican ${ }^{2}$.

Los resultados referentes a calidad metodológica (CM) en publicaciones del ámbito de la cirugía no han sido muy auspiciosos ${ }^{3-5}$; y es posible que en la esfera de la Cirugía Oral y Maxilofacial (COMF) este fenómeno sea similar. Es así como Lau y Samman $^{6}$, demostraron que ningún artículo en el área de la COMF publicado en revistas ISI durante los años 2002-2003 presentó un nivel de evidencia (NE) tipo 1 ; y que sólo el 2\% fue de NE 2. Así mismo, PitakArnnop et $\mathrm{al}^{7}$ y Sandhu ${ }^{8}$, reportaron el bajo número de ensayos clínicos publicados en revistas atingentes a esta especialidad.

Si bien se ha verificado el incremento de la productividad científica y la visibilidad internacional de la cirugía chilena en los últimos diez años ${ }^{9,10}$; a la fecha, no existen reportes relacionados con la cantidad y calidad de publicaciones en el área clínica de la COMF realizadas en Chile; lo que sin duda es relevante para una adecuada valoración de la generación de nuevo conocimiento para su aplicación en la práctica clínica ${ }^{4}$.

Por ello, el objetivo de este estudio es determinar la productividad y calidad metodológica $(\mathrm{CM})$ de artículos clínicos de COMF en Chile en el período 2001-2012.

\section{Material y Método}

\section{Diseño}

Estudio bibliométrico.

\section{Población a estudio}

Artículos relacionados a COMF generados por autores o instituciones chilenas en el período 20012012, años inclusive, bajo el tópico "cirugía oral maxilofacial", en revistas indizadas en las bases de datos: MEDLINE a través de su buscador PubMed, www.pubmed.org; "Science Citation Index Expanded" de Web of Science (Thomson-Reuters ex ISI) a través de la plataforma WoK www.webofknowledge. com; y SciELO, mediante su buscador en www. scielo.cl.

\section{Estrategias de búsqueda}

Las estrategias de búsqueda para cada una de las bases de datos consultadas, se detallan en la Tabla 1 . Todas éstas, fueron realizadas el 5 de enero de 2013.

\section{Maniobra}

Del resultado de la búsqueda, un investigador (PA) seleccionó mediante lectura de título y resumen, todos los artículos originales en los que al menos uno de sus autores tuviese filiación a una institución de salud, de investigación o entidad universitaria en Chile; y cuyos títulos y resúmenes presentaran temas clínicos atingentes a COMF según los parámetros descritos por la International Association of Oral and Maxillofacial Surgeons (IAOMS, http://www.iaoms.org/oms): Cirugía preprotésica; Cirugía oral y dentoalveolar y anestesia; Cirugía de implantes dentales y asociados a injerto óseo; Ciru- 
Tabla 1. Fuentes bibliográficas y estrategias de búsqueda de artículos clínicos en cirugía oral y maxilofacial (COMF) en Chile

\begin{tabular}{|c|c|c|}
\hline Base de datos & Estrategia de búsqueda & Límites de búsqueda \\
\hline MEDLINE & $\begin{array}{l}\text { Chile[AD] AND (Oral OR Maxillofacial) AND } \\
\text { (Surgery OR Surgical) NOT (Periodontol* OR endodon*) }\end{array}$ & $\begin{array}{l}\text { Filtros activos: Fecha de publicación entre } \\
\text { 01/01/2001 a 31/12/2012. Sólo revistas dentales }\end{array}$ \\
\hline $\begin{array}{l}\text { Web of } \\
\text { Science }^{\dagger}\end{array}$ & $\begin{array}{l}\text { SO = (J CRANIO MAXILL SURG OR BRIT J ORAL } \\
\text { MAX SURG OR INT J ORAL MAX SURG OR MED } \\
\text { ORAL PATOL ORAL OR J ORAL MAXIL SURG OR } \\
\text { J ORAL PATHOL MED OR ORAL MAXIL SURG } \\
\text { CLIN) AND CU = (Chile })\end{array}$ & $\begin{array}{l}\text { Tipo de documento }=(\text { Artículos }) \\
\text { Base de datos }=\text { SCI-EXPANDED tiempo de } \\
\text { búsqueda }=01-01-2011 \text { a 31-12-2012. Lemati- } \\
\text { zación = activa }\end{array}$ \\
\hline SciELO $^{\S}$ & $\begin{array}{l}\text { CIRUGÍA ORAL BUCAL MAXILOFACIAL } \\
\text { MAXILLOFACIAL SURGERY CHILE }\end{array}$ & $\begin{array}{l}\text { Fecha de publicación entre } 01 / 01 / 2001 \text { a } \\
31 / 12 / 2012 \text {. }\end{array}$ \\
\hline
\end{tabular}

†Búsqueda de artículos en las 5 revistas de cirugía oral y maxilofacial indizadas en la categoría "Dentistry, Oral Surgery and Medicine" de la herramienta "Journal Citation Report". "Uso de estrategia en barra de búsqueda disponible en www.scielo.cl

gía ortognática y deformidad dentofacial; Cirugía de trauma facial (fractura de huesos faciales y lesiones de tejidos blandos); Cirugía reconstructiva de cara; Cirugía de fisura labiopalatina; Deformidad congénita craneofacial; Cirugía plástica facial; Trastornos temporomandibulares; Cirugía correctiva de apnea del sueño; Cirugía de tumores y cánceres; y otros síndromes y manifestaciones orales de patologías sistémicas.

De los artículos seleccionados, dos investigadores (PA y RCV) realizaron una lectura en extenso de forma independiente, analizando y registrando las siguientes variables: Nombre de la revista; año de publicación; institución(es) o afiliación(es) del primer autor chileno; área quirúrgica clasificada según los parámetros de la IAOMS y los NE para terapia según propuesta de Sackett y cols ${ }^{11}$ (Tabla 2).

Posteriormente, y para determinar la CM de los artículos seleccionados, se aplicó la escala de medición MINCIR-terapia compuesta por 3 dominios y 6 ítems que utiliza los 18 puntos como punto de corte para definir el constructo $\mathrm{CM}^{12}$.

\section{Criterios de exclusión}

Se excluyeron todos aquellos artículos relacionados con otras áreas de la especialidad, como modelos de experimentación; biomecánicos y uso de elementos finitos; terapéutica no quirúrgica; análisis inmunohistoquímicos de patologías orales; estudios anatómicos, antropológicos, forenses de cabeza y cuello; y estudios de cirugía plástica no atingentes al territorio oral y máxilofacial. Se excluyeron además otro tipo de documentos, como: editoriales, notas técnicas, cartas al editor y revisiones narrativas de la literatura; así como también estudios de prevalencia y revisiones sistemáticas de la literatura. En situaciones de discrepancia en el registro de las variables y puntuación de CM, se llegó a un consenso evaluando en conjunto el documento.
Tabla 2. Clasificación de niveles de evidencia para artículos de terapia según Sackett y cols ${ }^{11}$

\begin{tabular}{|c|c|}
\hline Nivel & Tipo de estudio \\
\hline $1 \mathrm{a}$ & $\begin{array}{l}\text { Revisión sistemática de ensayos clínicos, } \\
\text { con meta-análisis }\end{array}$ \\
\hline $1 b$ & $\begin{array}{l}\text { Ensayo clínico aleatorio controlado individual } \\
\text { con intervalo de confianza estrecho }\end{array}$ \\
\hline $2 \mathrm{a}$ & Revisión sistemática de estudios de cohortes \\
\hline $2 b$ & $\begin{array}{l}\text { Estudios de cohortes individuales. Ensayo } \\
\text { clínico aleatorio de baja calidad }\end{array}$ \\
\hline $3 \mathrm{a}$ & $\begin{array}{l}\text { Revisión sistemática de estudios de casos y } \\
\text { controles }\end{array}$ \\
\hline $3 b$ & Estudio individual de casos y controles \\
\hline 4 & $\begin{array}{l}\text { Series de casos. Estudios de cohortes y de } \\
\text { casos y controles de mala calidad }\end{array}$ \\
\hline 5 & $\begin{array}{l}\text { Opinión de expertos sin evaluación crítica } \\
\text { explícita }\end{array}$ \\
\hline
\end{tabular}

\section{Plan de análisis}

Los datos fueron tabulados y analizados en el programa STATA 10,0. Se aplicó estadística descriptiva, con cálculo de porcentajes, promedios y su respectiva desviación estándar.

\section{Resultados}

Se identificaron 243 títulos a partir de las estrategias de búsqueda. La lectura de sus respectivos resúmenes permitió excluir en la base de datos SciELO 74 títulos por repetición o hipervínculos electrónicos sin acceso; 31 por corresponder a autores extranjeros y 23 por criterios de exclusión. En la base de datos MEDLINE, se eliminaron 17 artículos por criterios de exclusión; y en la Web of Science, 13 por replicación con la base de datos MEDLINE y 10 por 
criterios de exclusión. Posteriormente, en la lectura de texto completo, se excluyeron 6 artículos por carecer de datos relevantes. Por lo que el análisis final se realizó sobre una base de 68 artículos (Figura 1).

Se observó un aumento en el número de publica- ciones, desde $3(4,4 \%)$ el año 2002 a $8(11,7 \%)$ en el año 2012, hallándose un notorio incremento el año 2009 con 13 artículos (19,1\%) (Figura 2).

Según la institución primaria afiliada; el 80,9\% correspondía a universidades (Tabla 3).

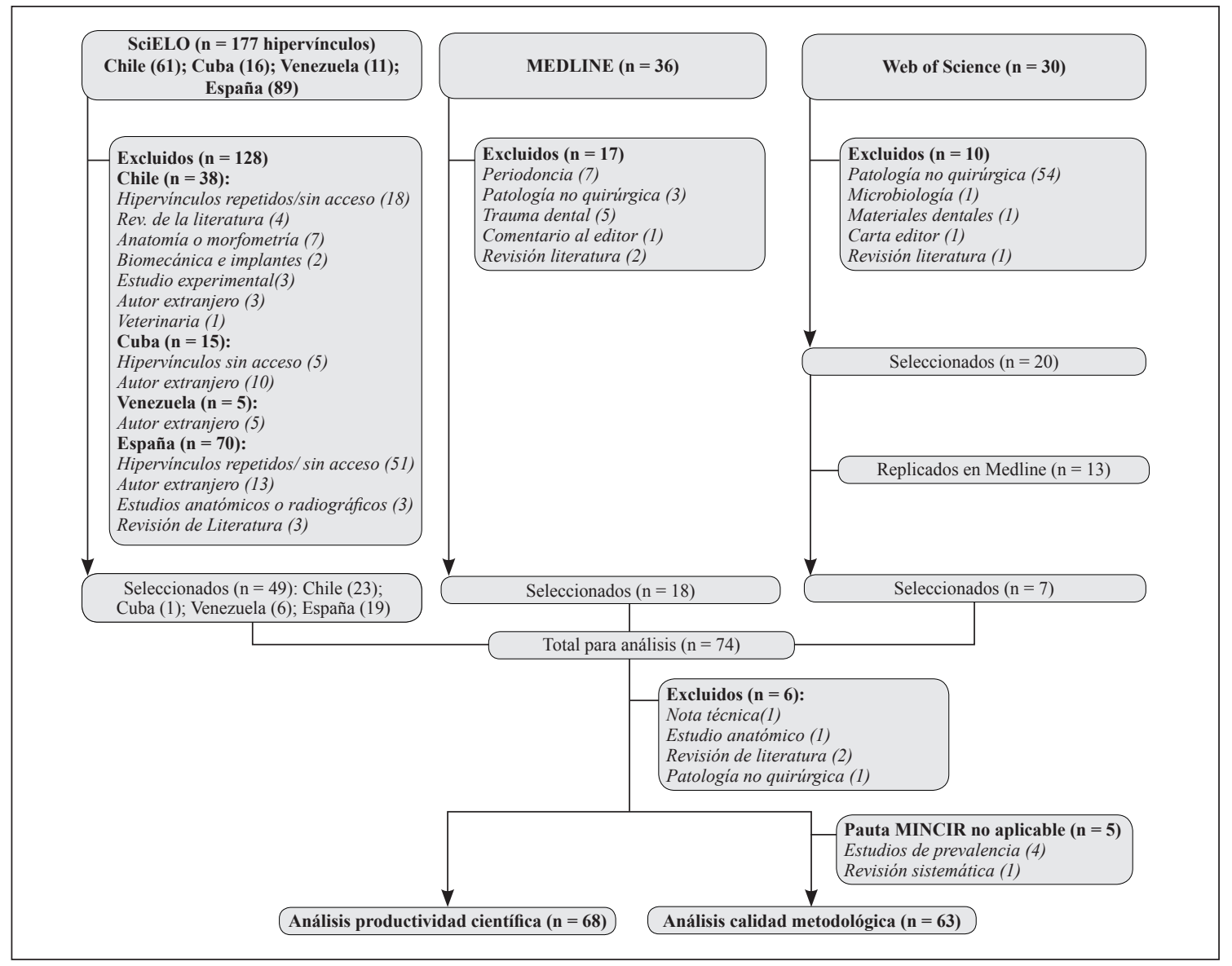

Figura 1. Flujograma de selección de artículos clínicos sobre COMF en Chile.

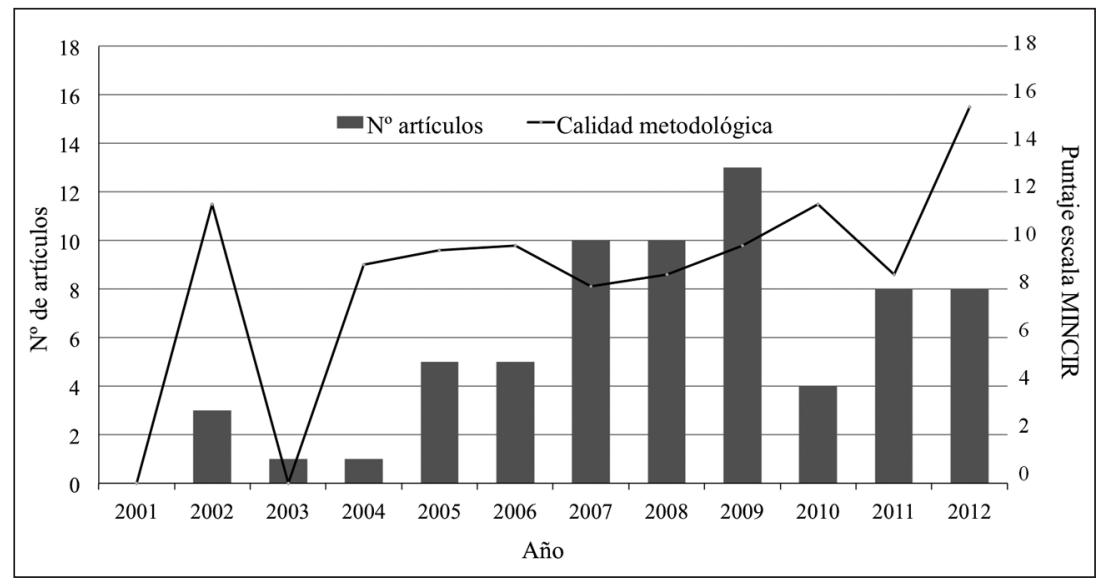

Figura 2. Productividad y valores promedios de $\mathrm{CM}$ de los artículos clínicos seleccionados de COMF-Chile según año en el período 2001-2012. 
Las revistas internacionales con mayor cantidad de publicaciones de autores chilenos son la Revista Española de Cirugía Oral y Maxilofacial con 12 artículos $(17,7 \%)$ y Medicina Oral Patología Oral y Cirugía Bucal con 11 artículos (16,2\%); y a nivel local la Revista Chilena de Cirugía y la Revista Médica de Chile con 4 artículos cada una (5,9\%) (Figura 3).

El $91,2 \%$ de los artículos analizados son de NE 4; el 7,4\% 2b y sólo un artículo 1a. La mayoría de los artículos corresponden a reporte y series de casos asociados a cirugía de tumores o cánceres (23 artículos; 33,8\%) (Tabla 4).

Tabla 3. Distribución de instituciones chilenas registradas en los artículos de COMF seleccionados

\begin{tabular}{|lcc|}
\hline Institución o afiliación & n artículos & \% \\
\hline Universidad de Chile & 20 & 29,4 \\
Universidad Mayor & 14 & 20,6 \\
\hline Universidad de La Frontera & 12 & 17,6 \\
\hline Universidad de Talca & 3 & 4,4 \\
\hline Hospital del Trabajador & 3 & 4,4 \\
\hline Clínica Alemana & 3 & 4,4 \\
\hline Universidad de Concepción & 2 & 2,9 \\
\hline Universidad Austral de Chile & 2 & 2,9 \\
\hline Hospital de Carabineros & 2 & 2,9 \\
\hline Otras instituciones * & 7 & 10,5 \\
\hline Total & 68 & 100 \\
\hline
\end{tabular}

*Pontificia Universidad Católica de Chile, Universidad Andrés Bello, Hospital Barros Luco, Hospital Mutual de Seguridad, Hospital del Salvador, Hospital San José y Hospital San Juan de Dios con un artículo cada cual.
El puntaje promedio de CM para todos los artículos analizados fue 9,9 \pm 4 puntos ( 6 a 27 puntos). Sólo 4 artículos superaron los 18 puntos. Se observó un incremento en la puntuación promedio de $\mathrm{CM}$ en los años $2010(11,6 \pm 2,4$ puntos $)$ y $2012(15,1 \pm 6,9$ puntos); lo que se puede apreciar en la Figura 2. No obstante ello, el promedio de los puntajes de CM según área quirúrgica se detalla en la Tabla 4.

\section{Discusión}

Según nuestros resultados, en Chile la COMF presenta un número promedio de 6 artículos por año; cifra que se puede considerar baja si se compara con la productividad disciplinaria de otros países en los que se generan entre 40 y 120 publicaciones anuales sólo en la base de datos ISI ${ }^{13}$. Esta situación, es similar a lo observado en otras disciplinas quirúrgicas a nivel nacional ${ }^{3}$. Sin embargo, y a pesar de ello, la tendencia a lo largo de la última década es positiva con un importante ascenso, similar a otras especialidades quirúrgicas ${ }^{9}$.

Se verificó una concentración de la producción en la Universidad de Chile y la Universidad Mayor, las que aportan el $50 \%$ de los artículos analizados. No obstante ello, cabe destacar la aportación de universidades regionales como la Universidad de La Frontera y la Universidad de Talca con un $17,6 \%$ y un $4,4 \%$ respectivamente, a expensas especialmente del último trienio.

Si bien estos resultados presentan sólo una tendencia, no debemos dejar de considerar algunas limitaciones del presente estudio que podrían implicar variaciones numéricas en dichos resultados: en primer lugar, las tres fuentes bibliográficas consultadas no cubren la totalidad de la productividad científica nacional; en segundo término, no existe

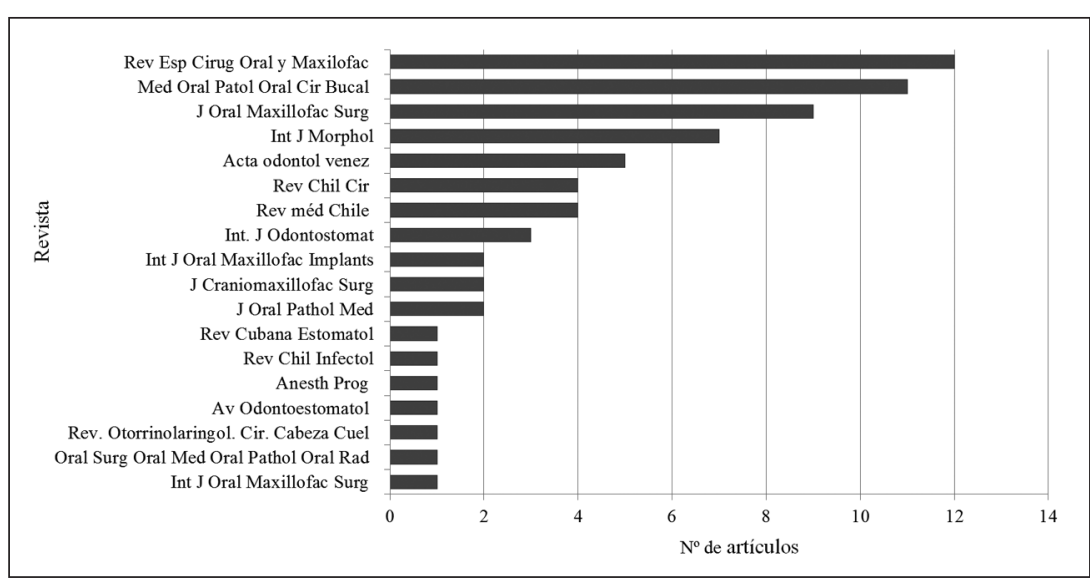

Figura 3. Nombre de revista de publicación de artículos chilenos de COMF 20012012 (según la abreviación utilizada en los buscadores electrónicos). 
Tabla 4. Nivel de evidencia y calidad metodológica MINCIR en áreas de COMF según IAOMS

\begin{tabular}{|lccccc|}
\hline & & \multicolumn{2}{c}{ NE Sackett } & \multicolumn{2}{c|}{$\begin{array}{c}\text { Escala MINCIR } \\
\text { Promedio } \pm \text { DE }\end{array}$} \\
Área quirúrgica COMF según IAOMS & Total (\%) & $\mathbf{1 a}$ & $\mathbf{2 b}$ & $\mathbf{4}$ & $\begin{array}{c}\text { Mromgía oral y dentoalveolar } \\
\text { Cirugín }\end{array}$ \\
Cirugía de implantes e injertos & $7(10,3)$ & 1 & 2 & 4 & $14,8 \pm 6,5$ \\
Cirugía ortognática & $6(8,8)$ & - & 1 & 5 & $11,2 \pm 3,8$ \\
Cirugía y trauma facial & $5(7,4)$ & - & 1 & 4 & $11,8 \pm 4,4$ \\
Cirugía reconstructiva de la cara & $10(14,7)$ & - & 1 & 9 & $12,6 \pm 4,7$ \\
Cirugía de fisura labiopalatina & $2(2,9)$ & - & - & 2 & $8,0 \pm 1,4$ \\
\hline Deformidad congénita cráneofacial & $3(4,4)$ & - & - & 3 & $10,0 \pm 1,4$ \\
Cirugía plástica facial & $1(1,5)$ & - & - & 1 & 8,0 \\
Trastornos temporomandibulares & $1(1,5)$ & - & - & 1 & 8,0 \\
Cirugía lesiones tumorales o cáncer & $1(1,5)$ & - & - & 1 & 7,0 \\
\hline Manifestación oral patologías sistémicas y síndromes & $9(13,2)$ & - & - & 9 & $7,7 \pm 1,6$ \\
\hline Total & $68(100,0)$ & 1 & 5 & 62 & $8,1 \pm 1,6$ \\
\hline
\end{tabular}

un algoritmo específico para determinar con certeza las publicaciones en el área de la COMF pudiendo haber quedado excluidos artículos atingentes a la especialidad debido a errores de indización ${ }^{14}$, cambios en el tiempo en los resultados de búsqueda debido a actualizaciones en las bases de datos ${ }^{14}$, o simplemente a variaciones en el registro de las afiliaciones de los autores chilenos adscritos en la publicación como entidad representativa.

Por otro lado, se ha de apreciar que la cantidad de publicaciones analizadas es escueta, por lo que pequeñas variaciones podrían tener un gran impacto en las cifras finales. Sin embargo, consideramos pertinente presentar estos resultados como el primer análisis de tendencias del número de artículos chilenos de COMF mediante una búsqueda sistemática exhaustiva y de acuerdo a la metodología expuesta.

En relación al NE, no es distinto al descrito a nivel mundial en las disciplinas quirúrgicas en general ${ }^{4}$ o en la COMF en particular-6; ${ }^{6-8}$ con una gran concentración a nivel de reporte de casos de patologías de baja prevalencia, hallazgos clínicos, complicaciones o resultados asociados a una técnica quirúrgica poco convencional. Si bien se observan ensayos clínicos dentro de los resultados, éstos carecen de conceptos metodológicos claros como: definición del diseño, estimación del tamaño de la muestra, asignación aleatoria de los grupos en estudio, presencia de enmascaramiento, etc.

Lo anterior implica un claro déficit en la generación de investigación clínica y aplicada de buen $\mathrm{NE}$; en especial en centros hospitalarios con equipos de formación de especialistas en COMF, las que trabajan con un alto volumen de casos y de mayor complejidad; pero quizás con escaso tiempo para la realización de investigaciones originales ${ }^{15}$.

Respecto a la CM de los artículos analizados, se verificó que ésta es baja; aunque con una tendencia a la mejoría en los últimos tres años representado en los 4 artículos con puntuación mayor a 18 puntos de la escala MINCIR-terapia ${ }^{16-19}$.

En general, todo esto implica que el aporte al conocimiento y desarrollo de la COMF en nuestro país es limitado y se acota básicamente a la publicación de artículos de NE tipo 4 de Sackett. Entre las estrategias que la literatura menciona como posibles caminos para mejorar esta situación en el campo quirúrgico, tenemos: exigir la realización de investigación asociada a publicaciones en revistas de corriente principal durante la obtención de la especialidad ${ }^{20}$; estimular la formación de Doctores en el grupo de especialistas ${ }^{20}$; implementar programas de formación de doble grado médico-odontológico en $\mathrm{COMF}^{21}$; y realizar cursos de escritura científica, reforzando el uso de pautas de escritura científica como CONSORT ${ }^{14}$, STROBE $^{23}$, PRISMA ${ }^{24}$, etc., dependiendo del tipo de estudio realizado.

En conclusión, el panorama de la producción científica en COMF en Chile ha mejorado en el último trienio, siendo aún escasa y de bajo NE y CM. Por ello, es perentorio desarrollar estrategias metodológicas tendientes a mejorar el volumen y calidad de la producción científica en esta área de la cirugía. 


\section{Agradecimientos}

Agradecemos al Dr. Julio Villanueva de la Unidad de Odontología Basada en Evidencia (OBE), de la Facultad de Medicina de la Universidad de Chile, por sus comentarios y aportes finales a este manuscrito.

La metodología de estudio y los resultados preliminares de este trabajo fueron presentados en la reunión científica de la Sociedad de Cirugía y Traumatología Bucal y Maxilofacial de Chile, el día 15 de marzo de 2012 en la ciudad de Santiago de Chile.

\section{Referencias}

1.- Vial M, Manterola C, Pineda. Revista Chilena de Cirugía: Índice de publicación según procedencia de los artículos. Rev Chil Cir. 2008;60:41-5.

2.- Spinak E. Diccionario Enciclopédico de Bibliometría, Cienciometría e Informetría. En: Caracas, UNESCO; 1996;34-131.

3.- Pineda V, Manterola C, Vial M, Losada H. ¿Cuál es la calidad metodológica de los artículos referentes a terapia publicados en la Revista Chilena de Cirugía? Rev Chil Cir. 2005;57:500-7.

4.- Manterola C, Pineda V, Vial M, Losada H, MINCIR Group. What is the methodologic quality of human therapy studies in ISI surgical publications? Ann Surg. 2006;244:827-32.

5.- Manterola C, Grande L. Methodological quality of articles on therapeutic procedures published in Cirugía Española. Evaluation of the period 2005-2008. Cir Esp. 2010;87:244-50.

6.- Lau S, Samman N. Levels of evidence and journal impact factor in oral and maxillofacial surgery. Int J Oral Maxillofac Surg. 2007;36:1-5.

7.- Pitak-Arnnop P, Sader R, Rapidis A, Dhanuthai K, Bauer U, Herve C, et al. Publication bias in oral and maxillofacial surgery journals: An observation on published controlled trials. J Cranio Maxillofac Surg. 2010;38:4-10.

8.- Sandhu A. The evidence base for oral and maxillofacial surgery: 10-year analysis of two journals. Br J Oral Maxillofac Surg. 2012;50:45-8.

9.- Moraga J, Cartes-Velásquez R, Manterola C, Urrutia $\mathrm{S}$. Publicaciones de autores chilenos en revistas quirúrgicas durante los últimos diez años. Rev Chil Cir. 2012;64:447-51.

10.- Cartes-Velásquez R, Moraga J, Aravena P, Manterola C. Impacto y visibilidad de la Revista Chilena de Cirugía tras su indización en las bases de datos SciELO e ISI: Análisis bibliométrico. Rev Chil Cir. 2012;64:511-5.

11.- Sackett DL, Wennberg JE: Choosing the best research design for each question. BMJ 1997; 315: 1636-40.

12.- Manterola C, Vial M, Pineda V, Sanhueza A. Systematic Review of Literature with Different Types of Designs. Int J Morphol. 2009;27:1179-86.

13.- Cartes-Velásquez R. Who publishes in leading Oral and Maxilllofacial Surgery Journals. Int J Oral Maxillofac Surg. 2011;40:1153.

14.- Costas R, Iribarren-Maestro I. Variations in content and format of ISI databases in their different versions. The case of the Science Citation Index in CD-ROM and the Web of Science. Scientometrics 2007;72:167-83.

15.- Mohammad A, Best A, Laskin D. Attitudes and opinions of residency directors and residents about the importance of research in oral and maxillofacial surgery residencies. J Oral Maxillofac Surg. 2011;69:2064-9.

16.- Andrades P, Borel C, Bartel R, Hernández R, Villalobos $\mathrm{R}$. Rendimiento de la evaluación clínica en el diagnóstico de fractura de huesos propios nasales. Rev Chil Cir. 2012;64:462-7.

17.- Halabí D, Escobar J, Muñoz C, Uribe S. Logistic regression analysis of risk factors for the development of alveolar osteitis. J Oral Maxillofac Surg. 2012;70:10404.

18.- Villanueva J, Araya I, Yanine N. Profilaxis antimicrobiana de corta duración versus profilaxis antimicrobiana de larga duración en cirugía maxilofacial mayor limpiacontaminada: Un estudio de cohorte. Rev Chil Infectol. 2012;29:14-8.

19.- Olate S, Lima Junior S, Sawazaki R, Moreira R, de Moraes M. Estudio descriptivo de los accesos quirúrgicos utilizados en el tratamiento de fracturas de complejo zigomático orbitario. Resultados preliminares. Int $\mathrm{J}$ Morphol. 2009;27:299-304.

20.- McKenchie A, McCaul J. Research training for oral and maxillofacial surgery. Br J Oral Maxillofac Surg. 2007;45:478-83.

21.- Cartes-Velásquez R, Ramírez H. Doble graduación en Cirugía Oral y Maxilofacial. Int J Odontostomat. 2011;5:191-8.

22.- Moher D, Schulz KF, Altman DG. The CONSORT statement: revised recommendations for improving the quality of reports of parallel-group randomised trials. Lancet 2001;357:1191-94.

23.- von Elm E, Altman DG, Egger M, Pocock SJ, Gøtzsche PC, Vandenbroucke JP; STROBE Initiative. The Strengthening the Reporting of Observational Studies in Epidemiology (STROBE) statement: guidelines for reporting observational studies. Ann Intern Med. 2007;147:573-77.

24.- Liberati A, Altman DG, Tetzlaff J, Mulrow C, Gøtzsche PC, Ioannidis JP, et al. The PRISMA statement for reporting systematic reviews and meta-analyses of studies that evaluate health care interventions: explanation and elaboration. J Clin Epidemiol. 2009;62:e1-e34. 